\title{
PRoPHET Using Optimal Path Hops
}

\author{
Salem Sati $^{\text {a, }}$, Tareg Abulifa ${ }^{\text {b }}$, Salah Shanab ${ }^{c}$ \\ ${ }^{a}$ Misurata University, IT Faculty, Misurata 218512, Libya \\ ${ }^{\mathrm{b}}$ The College of Industrial Technology, Computer Department, Misurata 218510, Libya \\ ${ }^{\mathrm{c}}$ The College of Industrial Technology, Computer Department, Misurata 218510, Libya \\ Email address: salem.sati@it.misuratau.edu.ly
}

Received: 27 January 2020; Accepted: 15 May 2020; Published: 08 August 2020

\begin{abstract}
The opportunistic network paradigm has no stable path and synchronous end-to-end communication. In such an environment, the bundle originator or the infected node stores a redundant copy in its buffer. This copy is replicated to other encountered susceptible nodes based on routing decision. The performance of the employed routing is based on delivering the bundle through optimal paths. This paper improves the decision criteria of the PRoPHET routing. This enhancement is achieved by employing the bundle probability and the optimal path hops. We have called our proposed improved PRoPHET utilizing Optimal Path Hops (PRoPHET-OPH). The evaluating of its performance is conducted by delivery probability, mean delay and percentage of overhead. Numerical findings exhibit that the PRoPHET-OPH performance is superior compared with the traditional PRoPHET.
\end{abstract}

Index Terms: Opportunistic Network, Prophet Performance, Evict Policy, Bundle Forwarding, Optimal Path.

\section{Introduction}

Opportunistic Network (OppNet) has not guaranteed end-to-end path. OppNet is consist of many wireless nodes. These nodes are opportunistically connected using a form of "Store-Carry-Forward". It consists of groups of nodes which form different regions. OppNets nodes are designed to connect in an environment characterized by high latency, high link distribution rate. This network has intermittent connectivity, in addition, it has no stable of the end-to-end route between both the source and destination of the bundle. Epidemic [1] is a common routing protocol in such network. The idea of the Epidemic is the bundle must copy to all encountered nodes which have a free buffer. Epidemic is powerful, but the memory of nodes is physically limited. Therefore, it consumes significant resources and its performance is degraded. To improve the performance of Epidemic, many researches suggested based on minimize the number of replicas, such as [2].To eliminate the problem of Epidemic, many routing has been suggested, such as Spray \& Wait [3] and PRoPHET [4]. The aim of those algorithms is to limit the bundle copies. These routing protocols make a decision criteria for copying the bundle. PRoPHET (Probability Routing Protocol with History of Encounters and Transitivity) uses delivery predictability (DP) between the nodes depending on their contact records, where higher delivery predictability indicates a greater probability of expected contacts. In addition, the bundle is transmitted to the met node only when forwarding judgment is satisfied. The bundle is transmitted when PRoPHET metric of the encountered node is greater than the current node. By arranging this, the PRoPHET algorithm performs a good delivery rate as well as it provides low overhead compared with Epidemic. Since it is transmitted only when a delivery predictability requirement is satisfied. This leads to insufficient bundle dissemination, high overhead ratio, and low delivery rate when the buffer capacity is insufficient. In this work, we enhance the distribution rate of the PRoPHET algorithm by employing ideal route hops [5] for propagating bundles on the entire network. The suggested replication is done if the two requirements of the forwarding strategy are satisfied. The first condition is the delivery predictability. The second is the bundle hops count less than the optimal path hops. Under both conditions; the node forwards the bundle to the encountered node. We observe that the suggested approach performs extraordinary delivery, low latency and overhead than the traditional PRoPHET. We enhance PRoPHET because it is a gradient protocol which is used by middlewares for opportunistic network such as IBR-DTN [6], and N4C [7]. We refer our introduced approach as PRoPHET using Optimal Path Hops (PRoPHET-OPH). The suggested protocol combines the advantages of delivery predictability and limiting the number of hops by selecting the optimal path to the destination. The parts sequence of this paper is as follows; related work is presented in section II. The suggested replication and evict policies is described in section III, while section IV explains the scenarios, model setting, experimental metrics and model result. Section V concludes the analyzed results and proposes future work. 


\section{Related Work}

PRoPHET uses delivery predictability metric to copy bundle to other nodes, PRoPHET minimizes the copies by selecting the node which has the significant delivery predictability value. There are a lot of researches consider a new approach to improve the PRoPHET performance such as PRoPHET+ [8], where the rate is computed as the summation of buffer size, cell power, position and status. Another approach by [9], where the DP is calculated based on the far between the couple nodes. In the paper [10], the authors suggest a regulated advanced approach for enhancing the performing effect of PRoPHET. The authors propose in their paper [11] an energy aware PRoPHET which considers the energy of the node and the available free buffer space. In [12] authors implement a framework termed DFP-DDP as a buffer management for improving PRoPHET performance. Moreover, the authors use All Hops Optimum Route (AHOP) [13] on the DTN network topology, this work taking into account two natures of weights to get the optimum route hops using real motion paths. Furthermore, the paper [5] analysis the problem of all hop optimal path as summation of the shortest path, in addition to the minimum/maximum for bottle neck approach.

\section{PRoPHET-OPH Routing Protocol}

The PRoPHET copies the bundle between linked nodes based on DP. When two nodes are encountered, the bundle is forwarded when the DP is high. We will propose the forwarding and evicting strategies for PRoPHET. Both strategies enhance the efficiency of PRoPHET .

\subsection{New Forward Strategy with Optimal Path Hops}

PRoPHET forwarding strategy determines when the bundle should be transmitted to the encountered node, this decision is made only if the encountered node has greater DP to destination. Now the open question is What is the value of this difference?. We have limited nodes as $\mathrm{N}$ and the whole of bundles copies Rc increased by one based on Eq. (1)

$$
R_{c}=1+H_{c}
$$

Where Rc is varying in 1 to max of [N-1], Hc the number of relayed nodes which the bundle is pass through. Referring to the efficiency analysis of opportunistic routing presented in [14], if Rc represent the copies of bundle which are disseminated in the network. Dt represent the remaining time to discard the bundle, the expected bundle delivery can be computed as

$$
P_{\exp }=1-e^{-D_{t} \lambda_{c} R_{c}}
$$

Where $\lambda_{c}$ is the disconnect event ratio, this ratio is the inverse of the average disconnect time between all mobile nodes, From Eq. (2), we can find the minimum value of the delivery probability as follow

$$
P_{1}=1-e^{-D_{t} \lambda_{c}} \approx D_{t} \lambda_{c}
$$

Furthermore, we can compute the forward decision criteria $F_{s}$ depends on bundle probability as minimum $F_{1}$ and optimal path hops $\mathrm{H}_{\mathrm{o}}$ as maximum $\mathrm{F}_{\mathrm{u}}$ by Eq. (4)

$$
F_{l}<F_{s}<F_{u}
$$

We can predict the relation between both of $\mathrm{H}_{\mathrm{o}}$ and $\mathrm{F}_{\mathrm{u}}$ which is considered as function of the delivery probability. From Eq. (3) and Eq. (4) we can formulate $F_{u}[13]$ as follow

$$
F_{u}=f\left(D P, H_{o}\right)=4 P_{1}
$$

Now we calculate $F_{1}$ for each forwarding decision, we compute our new forwarding strategy rule when two nodes linked each other. The minimum forwarding limit will be depend on $\mathrm{R}_{\mathrm{c}} \& \mathrm{H}_{\mathrm{c}}$ which related to the following

$$
F_{1}=D_{t} \lambda_{c}
$$


Finally we calculate both limits of encountered forward decision based on bundle probability and traditional metric DP. Similarly, our frontward approach has higher bound which computed based on the optimum route hop $\mathrm{H}_{\mathrm{o}}$ and minimum bound which computed as condition of the bundle probability.

\subsection{Evict Policy of PRoPHET-OPH}

PRoPHET-OPH determines its evict decision based on the bundle and the node information. Evict policy suggested to improve the performance of traditional PRoPHET such proposed by [15]. PRoPHET-OPH evict policy is designed as a utility function for each particular bundle in the network. The time and number of replications are used when a bundle will be evicted. This evict judgment is done by the node depending on bundle details. The evict moment which was predefined by the bundle creator in form of the discard time $\left(D_{t}\right)$ value. This time started since the bundle created. Essentially, copies of the bundle will be evicted when $\left(D_{t}=0\right)$ which means the bundle is expired. This impact by the current node spent time which is $\mathrm{B}_{\mathrm{t}}$. This rule which is employed by PRoPHET-OPH improves the performance as shown in Eq. (7). Additionally, to enhance the distribution probability, the scheme reduces the consumed energy by the bundle. The evict policy will remove the bundle which has a maximum evict function value $\mathrm{E}_{\mathrm{m}}$.

$$
E_{m}=D_{t}+B_{t} \cdot\left(R_{c}+1\right)+B_{t} \cdot\left(H_{c}+1\right)
$$

From Eq. (7), $R_{c}$ the number of copies that generated by the current node in the network. $H_{c}$ presents the number of passed nodes.

\section{Experiment and Results}

In order to evaluate PRoPHET-OPH, we compare PRoPHET-OPH with traditional PRoPHET using the performance metrics such as delivery probability, overhead ratio and delay.

\subsection{Scenarios and parameters}

The efficiency quality of PRoPHET-OPH is conducted by using the common ONE Simulator tool [16]. The evaluation and comparison of PRoPHET-OPH with traditional PRoPHET is shown in Table 1. As listed in the table 1., scenario 1 generates small bundles which similar to voice communication, where scenario 2 generates medium traffic load such as web browsing and best effort application. The third scenario generates large bundles like video applications. PRoPHET-OPH computes the path to the destination based on the bundle probability and the number of path hops. This concept improves the routing performance in such environment where commonly a number of optimal hops between 3 and 4 hops [13].

Table 1. Simulation Parameters

\begin{tabular}{|c|c|c|}
\hline No & Parameter & Value \\
\hline 1 & Simulation duration & $720 \mathrm{~min}$ \\
\hline 2 & Number of equipments & 126 \\
\hline 3 & Set Type with Velocity & $\begin{array}{c}80 \text { Hand held }(0.5-1.5 \mathrm{~km} / \mathrm{h}) \\
40 \text { Taxi }(10-70 \mathrm{~km} / \mathrm{h}) \\
6 \text { Buses }(10-90 \mathrm{~km} / \mathrm{h})\end{array}$ \\
\hline 4 & Movement zone & $3400 \times 4500 \mathrm{~m}^{2}$ \\
\hline 5 & Data Delivery Rule & PRoPHET\& PRoPHET-OPH \\
\hline 6 & Card type & Simple one-to-all \\
\hline 7 & Radio Coverage & $0.25 \mathrm{~km}$ \\
\hline 8 & Bandwidth & $0.25 \mathrm{Mbps}$ \\
\hline 9 & Evict policies & DF, MOFO, SHLI \\
\hline 10 & Bundle size & $\begin{array}{c}\text { Scenario 1: } 500-1024 \mathrm{~KB} \\
\text { Scenario 2: } 64-512 \mathrm{~KB} \\
\text { Scenario 3: } 1-5 \mathrm{MB} \\
\end{array}$ \\
\hline 11 & Bundle creation period & $\begin{array}{l}\text { Scenario } 1: 20-30 \mathrm{~s} \\
\text { Scenario 2: 45-50s } \\
\text { Scenario 3: } 1-2 \mathrm{~min} \\
\end{array}$ \\
\hline 12 & Time-to-live (TTL) & $100-500$ step $100 \mathrm{~min}$ \\
\hline 13 & Default storage size & $\begin{array}{l}\text { Hand Held: } 5 \mathrm{MB} \\
\text { Taxi, Buses: } 50 \mathrm{MB}\end{array}$ \\
\hline
\end{tabular}

\subsection{Numerical Results}

Our evaluation is shown in fig.1. for the different scenarios. We compare PRoPHET-OPH with traditional PRoPHET which applies different evict policies. We observe from the fig.1. that only the PRoPHET-OPH has higher delivery probability. Traditional PRoPHET deploys different policies which are Drop Front (DF), MOst FOrwarded (MOFO), and SHort LIfe (SHLI). PRoPHET-OPH is better than traditional PRoPHET with MOFO in fig.1.d and fig.1.g about $10 \%$. We can also observe from fig.1.a that the difference reaches 3\% when PRoPHET using MOFO. In fig.1.a, 
we can find that percentage difference between PRoPHET-OPH and traditional PRoPHET using MOFO is between 13\%. In fig.1.a, PRoPHET using DF policy has low delivery of $10 \%$ in comparison with the PRoPHET-OPH routing. Commonly, the average variant in the bundle handling ratio between PRoPHET-OPH and traditional PRoPHET is low because the generated traffic is small. The delivery ratios are rather low here when compared with PRoPHET using MOFO and SHLI. This is due to the low mobility of the different nodes. But it may give another view when we compare the delivery ratio to overhead as resource consumption metric. The idea of PRoPHET-OPH routing targets to remove the unnecessary bundles copies of Epidemic. PRoPHET-OPH archives this by deploying the optimal end-to-end hops as upper forwarding limit. We got quite improving in delivery rate. Moreover, we save a lot of resources (by reducing overhead). Therefore, as fig.1.b,e and h show that the number of transmitted bundles for PRoPHET-OPH are the lower compared with traditional PRoPHET. This is due to that PRoPHET-OPH has the ability to select the optimum and shortest path. This can be seen in both decisions of forward and evict regardless of the increase in traffic load. Here it is worthwhile to mention to overhead, PRoPHET-OPH has the minimum overhead. Traditional PRoPHET using SHLI is the worst energy consumption specially in fig.1.b and h. Fig.1.b illustrates that the overhead of traditional PRoPHET using SHLI grows sharply when TTL is greater than $200 \mathrm{~min}$. In the three mentioned overhead figures, we notice that with increased TTL of the bundle, both policies of DF and MOFO are increased.

In fig.1.b with TTL=500 min, traditional PRoPHET using SHLI has a high traffic overhead up to 380\%. In addition, traditional PRoPHET using MOFO produces an overhead of 38\%, while PRoPHET-OPH routing remains throughout different three scenarios and TTL variations at 2\% to 15\%. Traditional PRoPHET using MOFO produces $20 \%$ greater overhead than PRoPHET-OPH. The overhead ratio of PRoPHET-OPH is always less than traditional PRoPHET using different evict policies at the level of around 20\%. This is because PRoPHET-OPH considers the overhead with the optimal path hops. Furthermore, PRoPHET-OPH improves the buffer management by accurate final forwarding and dropping decisions. The forward strategy considers the forward error using the optimal path hop. The optimal path hop improves PRoPHET-OPH decision which eliminates unnecessary copies to minimize the relayed bundles. Traditional PRoPHET using SHLI does not consider the overhead. This is because it uses the TTL as decision criteria for SHLI evict policy. However, MOFO policy produces less overhead than SHLI. This is due to that MOFO decision depends on the hop and replication counts, while SHLI policy depends on bundle lifetime. Therefore, MOFO still has a problem of delay. The delay with varying TTL and traffic are shown in fig.1.c,f and i. The figures show that the bundle delay increases as TTL increases in all three scenarios. In fig.1.c, traditional PRoPHET using SHLI and DF leads to the closest average delay to PRoPHET-OPH delay, but the PRoPHET- OPH routing has less delay of around 400ms. For fig.1.c,f and i PRoPHET-OPH generates fewer delay values. The enhancement of PRoPHET-OPH regarding to the minimum delay due to the advantage of optimal path. For fig.1.c,f and i, traditional PRoPHET using MOFO policy has the worst delay when it compared with other evict policies, where this policy does not consider any time. The delay of MOFO varies as TTLs axis varies from 100 to 500 by step $100 \mathrm{~min}$. Traditional PRoPHET using MOFO has higher delays ranging from 300 to $2000 \mathrm{~ms}$ more than PRoPHET-OPH routing.

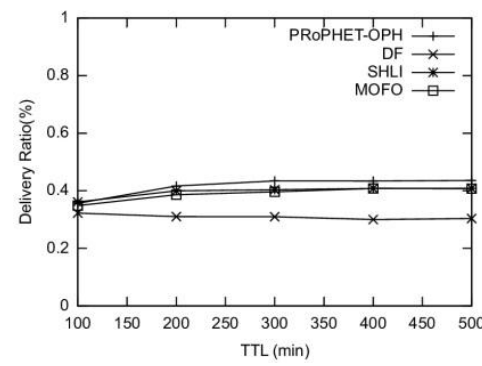

(a) Scenario 1: Delivery Ratio

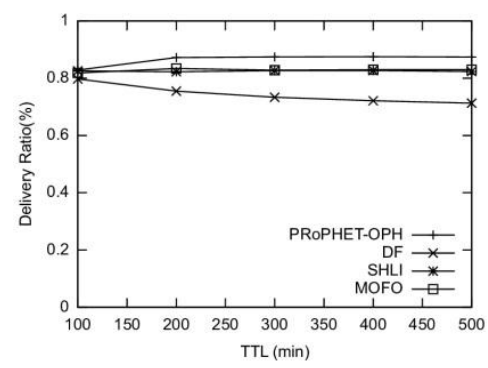

(d) Scenario 2: Delivery Ratio

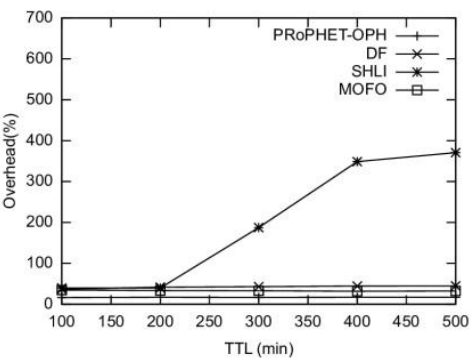

(b) Scenario 1: Overhead

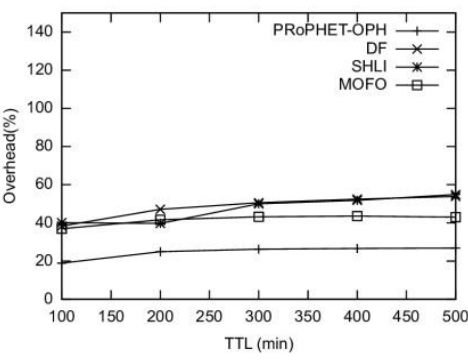

(e) Scenario 2: Overhead

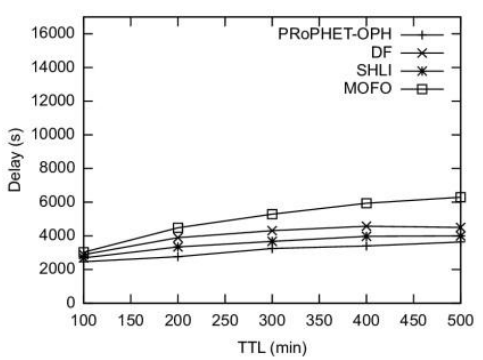

(c) Scenario 1: Delay

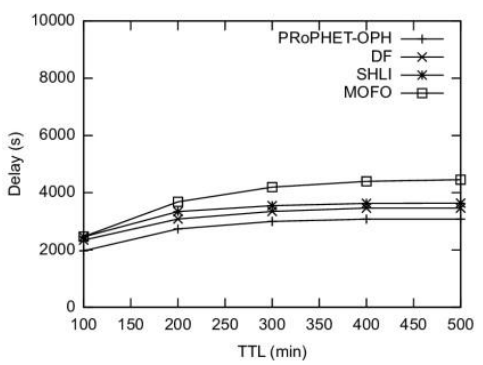

(f) Scenario 2: Delay 


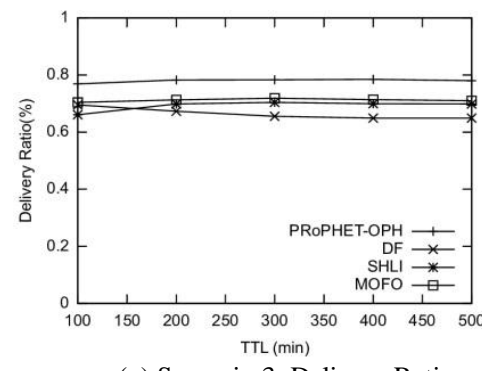

(g) Scenario 3: Delivery Ratio

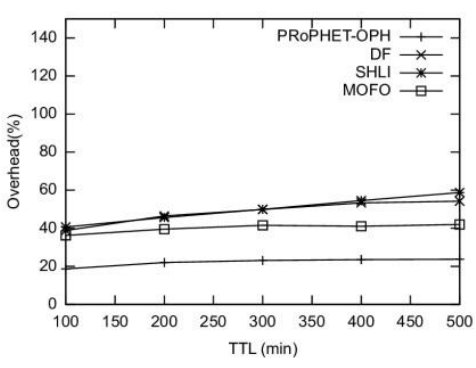

(h) Scenario 3: Overhead

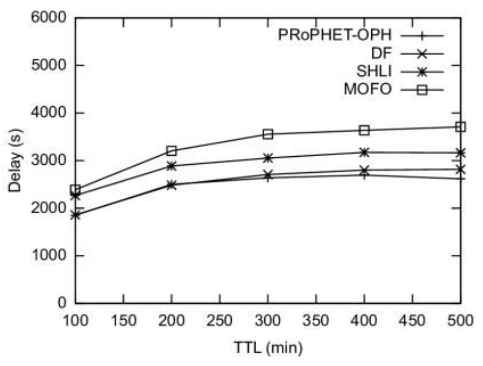

(i) Scenario 3: Delay

Fig.1. Result of proposed PRoPHET-OPH and traditional PRoPHET

\subsection{Summary}

Form the results of different scenarios, we found that the forwarding decision of PRoPHET-OPH is accurate. The forwarding decision is based on delivery probability and optimal path hope as described in Eq.(4). In addition, we deploy evict policy by Eq.(7) to improve the performance of PRoPHET-OPH, while traditional PRoPHET uses forwarding decision based on delivery probability only. We apply DF, MOFO and SHLI as evict policies for traditional PRoPHET. The results show that PRoPHET-OPH has better delivery ratio compared with traditional PRoPHET. Furthermore, PRoPHET-OPH has minimum overhead and delay. This means that PRoPHET-OPH concentrates the most challenges of opportunistic nodes which are storage and energy.

\section{Conclusion and Future Work}

PRoPHET is one of the important opportunistic routing protocols. PRoPHET is implemented in reality for opportunistic middleware. The performance of traditional PRoPHET routing needs to enhancement because it calculates its metric based on delivery probability. This paper improves the traditional PRoPHET by suggesting a new opportunistic routing protocol. We called it as PRoPHET-OPH. This PRoPHET-OPH considers the optimal path hop in addition to delivery probability. PRoPHET-OPH approach considers the path length as routing weight to eliminate the extra bundles copies. This advanced strategy reduces the power consumption in such an environment. Results gathered from the simulation scenario show that PRoPHET-OPH performance is improved in delivery ratio. The results show that PRoPHET-OPH has lower overhead of about $25 \%$ in all different scenarios while traditional PRoPHET produces $40 \%$ overhead as a minimum overhead for different scenarios. This means that PRoPHET-OPH save the energy and storage of the node when compared with traditional PRoPHET overhead. Furthermore, PRoPHET-OPH gives good quality of service when compared with delay of traditional PRoPHET.

As future work, we plan to investigate and analyze the social based PRoPHET forwarding by laying a focus on user mobility and relationships. Social forwarding depends on People-Rank as routing metric. This social interaction may help to take the accurate forwarding decision in such an opportunistic network. We suggest People-Rank using a calculated weight based on social and contact information.

\section{References}

[1] A. Vahdat, D. Becker et al., "Epidemic Routing for Partially Connected Ad hoc Networks," Technical Report CS-200006, Duke University, Tech. Rep., April 2000.

[2] S. Sati, A. Sohoub, A. Eltahar, K. A. B. Ahmad, K. Ahmad, and A. Bakeer, "Degree Contact DC-Epidemic Routing Protocol For Opportunistic Networks," in Proceedings of Advances in Wireless and Optical Communications (RTUWO), pp. 198-203, November 15-16, 2018, Riga, Latvia, 2018.

[3] T. Spyropoulos and K. Psounis, "Spray and Wait: An Efficient Routing Scheme for Intermittently Connected Mobile Networks ," Proceedings of the ACM SIGCOMM Workshop on Delay-Tolerant Networking, , 2005.

[4] A. Lindgren, A. Doria, E. Davies, and S. Grasic, "RFC 6693: Probabilistic Routing Protocol for Intermittently Connected Networks," IETF, 2012.

[5] R. Gu'erin and A. Orda, "Computing shortest paths for any number of hops," IEEE/ACM Trans. On Netw., vol. 10, no. 5, pp. 613-620, 2002.

[6] M. Doering, S. Lahde, J. Morgenroth, and L. C. Wolf, "IBR-DTN: an efficient implementation for embedded systems," in Proceedings of the Third Workshop on Challenged Networks, CHANTS 2008, San Francisco, 2008, pp. 117-120, California, USA, September 15, 2008.

[7] N4C, "Functional specification for dtn infrastructure software," 2008. [Online]. Available: http://www.n4c.eu/N4C-info.php

[8] T. Huang, C. Lee, and L. Chen, "Prophet+: An adaptive prophet-based routing protocol for opportunistic network," in 24th IEEE International Conference on Advanced Information Networking and Applications, AINA 2010, pp. 112-119, Perth, Australia, 20-13 April 2010, 2010.

[9] P. Sok and K. Kim, "Distance-based prophet routing protocol in disruption tolerant network," in Proceedings of the 
International Conference on ICT Convergence (ICTC '13), pp. 159-164, Jeju , Korea, October 2013.

[10] A. E. Ouadrhiri, M. El-Kamili, M. R. El-Fenni, and L. Omari, "New forwarding strategy for PRoPHET routing in delay tolerant networks" in Networked Systems - First International Conference, NETYS 2013, pp. 300-305, Marrakech, Morocco, May 2-4, 2013, Revised Selected Papers, 2013.

[11] B. B. Bista and D. B. Rawat, "Ea-PRoPHET: An energy aware PRoPHET based routing protocol for delay tolerant networks," in 31st IEEE International Conference on Advanced Information Networking and Applications, AINA 2017, pp. 670-677, Taipei, Taiwan, March 27-29, 2017, 2017.

[12] S. Sati, C. Probst, and K. Graffi, "Implementing forward and drop policies for improving PRoPHET's routing performance," in 12th International Conference on Mobile Ad-Hoc and Sensor Networks, MSN 2016, pp. 236-242, Hefei, China, December 1618, 2016, 2016.

[13] S. Bayhan, E. Hyyti"a, J. Kangasharju, and J. Ott, "Analysis of hop limit in opportunistic networks by static and timeaggregated graphs," in 2015 IEEE International Conference on Communications, ICC 2015, pp. 3287-3292, London, United Kingdom, June 8-12, 2015, 2015.

[14] E. Bulut, Z. Wang, and B. K. Szymanski, "Cost-effective multi period spraying for routing in delay-tolerant networks," IEEE/ACM Trans. Netw., vol. 18, no. 5, pp. 1530-1543, 2010.

[15] S. Sati, K. Ahmad, "Efficient evict policy for PRoPHET". International Journal of Information Technology. vol 12, issues 1, pp 251-260 (2020). https://doi.org/10.1007/s41870-019-00359-x.

[16] A. Ker"anen, J. Ott, and T. K"arkk"ainen, "The ONE Simulator for DTN Protocol Evaluation," in Proceedings of the International Conference on Simulation Tools and Techniques for Communications, Networks and Systems (SimuTools). ICST/ACM, 2009.

\section{Authors' Profiles}

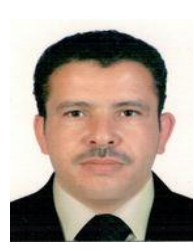

Salem Sati has a PhD in computer science from HHU University in Dusseldorf, Germany (2017), and an MSc \& BSc degree in computer engineering from Higher Industrial Institute in Misurata, Libya (2008 \& 1997 , respectivally).. Dr. Sati has contributed to several national conferences in Libya, Also International IEEE conferences in North America. Asia and Europe in the areas of computer networks. He has many publications in IEEE conferences. He is currently a Doctor at the faculty of Information Technology in Misurata University -Libya.

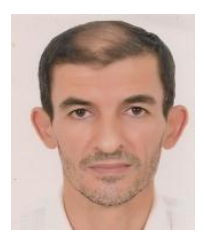

Tareg Abulifa has MSc degree in Electrical and electronic engineering from Glasgow University, Scottland (2011), and BSc degree in computer Engineering from Higher Industrial Institute in Misurata (1995). He is the head of the technical support department at The Collage of Industrial Technology, Misurata. He has many publications in several local and international conferences and jornals.

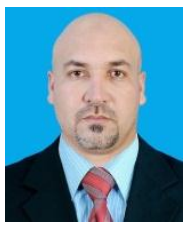

Salah Shanab is a lecturer at the department of Electronic Engineering in The Collage of Industrial Technology, Misurata. He had a BSc degree in computer Engineering from Higher Industrial Institute, Libya (2000). and an MSc degree in computer engineering from Academy of Graduate Studies, Libya (2013).

How to cite this paper: Salem Sati, Tareg Abulifa, Salah Shanab, "PRoPHET Using Optimal Path Hops", International Journal of Wireless and Microwave Technologies(IJWMT), Vol.10, No.4, pp. 16-21, 2020.DOI: 10.5815/ijwmt.2020.04.02 